\title{
Characteristics of Graduating Family Medicine Residents Who Intend to Practice Maternity Care
}

Sebastian T. Tong, MD, MPH; Camille J. Hochheimer; Wendy B. Barr, MD, MPH; Matteo Leveroni-Calvi, MD; Nicholas M. Lefevre, MD; Jordyn T. Wallenborn, PhD, MPH; Lars E. Peterson, MD, PhD

\begin{abstract}
BACKGROUND AND OBJECTIVES: Prior research has found that $24 \%$ of graduating family medicine residents intend to provide obstetrical deliveries, but only $9 \%$ of family physicians 1 to 10 years into practice are doing so. Our study aims to describe the individual and residency program characteristics associated with intention to provide obstetrical deliveries and prenatal care.

METHODS: Cross-sectional data on 2014 to 2016 graduating residents were obtained from the American Board of Family Medicine certification examination demographic questionnaire that asked about intended provision of specific clinical activities. A hierarchical model accounting for clustering within residency programs was used to determine associations between intended provision of maternity care with individual and residency program characteristics.
\end{abstract}

RESULTS: Of 9,541 graduating residents, $22.7 \%$ intended to provide deliveries and $51.2 \%$ intended to provide prenatal care. Individual characteristics associated with a higher likelihood of providing deliveries included female gender, graduation from an allopathic medical school, and participation in a loan repayment program. Residency characteristics included geographic location in the Midwest or West regions, training at a federally qualified health center (FQHC)-based clinic, funding as a teaching health center (THC), more months of required maternity care rotations, larger residency class size, and maternity care fellowship at residency.

CONCLUSIONS: Our findings suggest that increasing the proportion of graduating family medicine residents who intend to provide maternity care may be associated with increased exposure to maternity care training, more family medicine training programs in FQHCs and THCs, and expanded loan repayment programs.

(Fam Med. 2018;50(5):345-52.) doi: 10.22454/FamMed.2018.631796

Published Online First March 8, 2018

amily physicians are trained to provide comprehensive care, ${ }^{1,2}$ and providing this full spectrum of care is associated with lower total costs and reduced hospitalizations. ${ }^{3}$ Despite this, family physicians are decreasing their scope of practice. $^{4-7}$ This decrease in scope has been particularly noted in maternity care with provision by family physicians declining from $23.3 \%$ in 2000 to $9.7 \%$ in $2010 .{ }^{6}$ However, a recent study using 2014 survey data from the American Board of Family Medicine (ABFM) at the time of application for board certification suggests that more graduating residents report intention to provide maternity care than that reported by current family physicians. ${ }^{8,9}$ Specifically, a cross-sectional study found that while $23 \%$ of graduating family medicine residents reported intention to provide obstetrical deliveries when applying for initial certification, $9 \%$ reported actual provision of deliveries when recertifying 1 to 10 years into practice. Similarly, $50 \%$ of graduating residents report intent to provide prenatal care, but only $12 \%$ report doing so 1 to 10 years into practice.

The recent Accreditation Council for Graduate Medical Education (ACGME) change to eliminate minimum obstetrical delivery requirements in family medicine training requirements has caused concern about the quality of maternity care education. ${ }^{10} \mathrm{~A}$ recent family medicine residency program director survey reported highly variable maternity care experiences in residency training and found certain residency characteristics were associated with increased graduate practice of maternity care, including

From the Virginia Commonwealth University School of Medicine (Drs Tong and Wallenborn, and Ms Hochheimer); the Lawrence Family Medicine Residency and the Tufts University School of Medicine (Drs Barr, LeveroniCalvi, and Lefevre); and the American Board of Family Medicine and the University of Kentucky College of Medicine (Dr Peterson). 
community-based, university-affiliated programs and regional location in the Midwest and West. ${ }^{11}$ However, these results were reported by program directors and could be subject to bias. Improving the understanding of residency-level characteristics associated with intention to practice maternity care, as reported by individual family medicine residency graduates, may help policy efforts to enable family physicians to practice maternity care.

Our study aimed to describe the associations between both characteristics of family medicine residents and residency programs with intent to provide obstetrical deliveries and prenatal care near the end of residency when applying for initial ABFM certification.

\section{Methods}

Using a cross-sectional study design, we completed a secondary analysis of data collected from physicians applying for initial ABFM certification in 2014-2016. The Virginia Commonwealth University Institutional Review Board approved this study as exempt research.

\section{Data Collection}

The data were collected at the initiation of application for the Family Medicine Certification examination, which for graduating residents usually takes place midway through their last year of residency or just after residency. Questions included demographic information, future practice characteristics and organization (if known), and intended scope of practice. Specifically, applicants were asked if they intended to provide obstetrical deliveries and practice prenatal care. Completion of the questionnaire was a required component of the application, and yielded a $100 \%$ response rate. Individual characteristics were self-reported by respondents and are reported in our study as the questions were asked in the ABFM questionnaire, the details of which are shown in Table 1 of Coutinho et al. ${ }^{8}$ We linked applicants to their residency programs to explore the associations of residency-level characteristics with intentions to provide maternity care. Residency program characteristics were primarily obtained from the American Academy of Family Physicians (AAFP) Residency Directory ${ }^{12}$ and supplemented with the Teaching Health Center (THC) directory ${ }^{13}$ and the rural training track directory. ${ }^{14}$ Residencies were classified to regions based on the US Census Bureau regional definitions..$^{15} \mathrm{We}$ matched $99.2 \%$ of graduating residents to their residency program; however, some AAFP Residency Directory data were missing.

\section{Data Analysis}

We used descriptive statistics to characterize the study sample overall as well as by intention to practice obstetrical deliveries or prenatal care. A hierarchical model accounting for clustering within residencies was used to examine both the univariate and multivariate association between intention to provide obstetrical deliveries or prenatal care and individual and residency characteristics. All characteristics with a significant univariate association with the outcome of interest were entered into a multivariate model, and all significant odds ratios and corresponding $95 \%$ confidence intervals from the multivariate model were reported. These analyses were completed using SAS version 9.4 (Cary, NC). Then, using $R$ version 3.3.0 (Vienna, Austria), frequencies were mapped at the state level using the rgdal, maptools, mapproj, rgeos, and $s p$ packages.

\section{Results}

Our sample included a total of 9,541 graduating family medicine residents who applied for initial ABFM certification from 2014-2016 (Table 1). In this sample, $55.1 \%$ were female, $80.9 \%$ were allopathic medical school graduates, $19.0 \%$ participated in a loan repayment program, and the mean age was 32.9 years. Of all residents in the sample, $22.7 \%$ reported intention to perform obstetrical deliveries, and $51.2 \%$ reported intention to provide prenatal care.

In univariate analysis, individual characteristics associated with intent to provide obstetric deliveries included being female, graduating from an allopathic medical school, having moderate educational debt (between $\$ 25,000-\$ 149,999$ vs less than $\$ 25,000$ or more than $\$ 150,000$ ) and participating in a loan repayment program (Table 2). Except for educational debt, these associations held for intent to practice prenatal care. Residency characteristics associated with both intent to provide deliveries and prenatal care were funding as a THC, training based in a federally qualified health center (FQHC), increased months of required maternity care rotations, presence of a maternity care fellowship at the residency program, and larger residency class size (Table 2). Increased number of beds and the presence of a rural training track were associated with intent to provide deliveries but not prenatal care. In addition, regional patterns existed with higher proportions of graduating residents from residencies in the Midwest or West regions intending to provide both obstetric deliveries and prenatal care. This proportion ranged from $3.9 \%$ of graduating residents training in Mississippi intending to provide obstetric deliveries to $70.3 \%$ in Alaska (Figure 1). Similarly, prenatal care intent ranged from $27.3 \%$ in West Virginia to $83.8 \%$ in Alaska (Figure 2).

In the adjusted multivariate analysis, individual characteristics found to be associated with intent to provide obstetrical deliveries were female gender, being an allopathic medical school graduate, and participation in a loan repayment program. The same individual variables were associated with intent to provide prenatal care (Table 3 ). Residency characteristics associated with intent to provide deliveries were geographic location, increased months of required maternity care rotations, presence of a maternity care fellowship, funding as a THC, and lower 
number of beds at primary admitting hospital. No statistically significant relationship was found for training in a FQHC. Residency characteristics associated with intent to provide prenatal care were geographic location, increased months of required maternity care rotations, training in a FQHC, and larger residency class size. Statistically significant relationships were not found between funding as a FQHC and prenatal care provision intent or between having a maternity care fellowship and prenatal care provision intent.

\section{Discussion}

Our study of every graduating family medicine resident seeking board certification for 3 consecutive years found multiple individual and residency program-level characteristics associated with higher likelihood of intending to practice maternity care. In addition to strong geographic variation, we found that more required months in maternity care rotations and participation in loan repayment programs were associated with higher intentions to provide obstetrical deliveries and prenatal care. Furthermore, training at a FQHC or THC-funded program was associated with increased prenatal care or delivery provision intent, respectively.

Notably, the only nongeographic, residency-level variable associated with both increased intent to provide deliveries and prenatal care was increased months of required maternity care rotation. While residents who do not want to provide maternity care may self-select into programs with fewer maternity care curricular requirements, a strength of this study is that we controlled for this by including months of required maternity care rotations into our multivariate model. If family medicine as a specialty values the continued provision of maternity care, efforts may need to be taken to encourage residency programs to require increased maternity care exposure. In order to increase maternity care in residencies, guidance may need to be provided to residency programs that struggle to maintain existing maternity care curricula due to logistical barriers.

\section{Limitations}

Several limitations exist for this study. First, practice choices may be fluid at the time of application for Family Medicine Certification Examination, which usually takes place midway through the last year of training. Second, the cross-sectional study design cannot determine causation, and there may be other residency characteristics or curricular

Table 1: Summary of Family Medicine Resident and Residency Program Characteristics

\begin{tabular}{|l|c|}
\hline \multicolumn{1}{|c|}{ Characteristic Individual Characteristics (N=9,541) } \\
\hline Gender & Number (\%) or Mean (SD) \\
\hline Male & $4,284(44.9 \%)$ \\
\hline Female & $5,257(55.1 \%)$ \\
\hline Medical School Type & $1,818(19.1 \%)$ \\
\hline Osteopathic & $7,723(80.9 \%)$ \\
\hline Allopathic & $32.9(4.4)$ \\
\hline Age (in years) & $1,969(20.6 \%)$ \\
\hline Educational Debt* & $1,716(18.0 \%)$ \\
\hline Less than $\$ 25,000$ & $2,927(30.7 \%)$ \\
\hline$\$ 25,000-\$ 149,999$ & $2,929(30.7 \%)$ \\
\hline$\$ 150,000-\$ 249,999$ & $1,814(19.0 \%)$ \\
\hline$\$ 250,000$ or greater & $7,727(81.0 \%)$ \\
\hline Loan Repayment Program Participation** & \\
\hline Yes & \\
\hline No & $1,660(17.5 \%)$ \\
\hline \multicolumn{2}{|c|}{ Residency Characteristics by Resident } \\
\hline Geographic Region*** & $3,049(32.2 \%)$ \\
\hline Northeast & $2,684(28.4 \%)$ \\
\hline South & $2,068(21.9 \%)$ \\
\hline Midwest & \\
\hline West & \\
\hline
\end{tabular}


Table 1, continued

\begin{tabular}{|c|c|}
\hline \multicolumn{2}{|c|}{ Residency Characteristics by Resident } \\
\hline Characteristic & Number (\%) or Mean (SD) \\
\hline \multicolumn{2}{|c|}{ Residency Funding as Teaching Health Center } \\
\hline Yes & $382(4.0 \%)$ \\
\hline No & $9,079(96.0 \%)$ \\
\hline \multicolumn{2}{|l|}{ Federally Qualified Health Center-Based } \\
\hline Yes & $1,866(21.2 \%)$ \\
\hline No & $6,933(78.8 \%)$ \\
\hline \multicolumn{2}{|l|}{ Rural Training Track at Residency } \\
\hline Yes & $815(8.6 \%)$ \\
\hline No & $8,646(91.4 \%)$ \\
\hline \multicolumn{2}{|l|}{ Months of Required Obstetrics Rotations } \\
\hline 2 & $3,928(44.8 \%)$ \\
\hline 3 & $2,496(28.5 \%)$ \\
\hline 4 & $1,675(19.1 \%)$ \\
\hline 5 & $345(3.9 \%)$ \\
\hline 6 & $327(3.7 \%)$ \\
\hline \multicolumn{2}{|l|}{ Obstetrics Fellowship at Residency } \\
\hline Yes & $946(10.7 \%)$ \\
\hline No & $7,907(89.3 \%)$ \\
\hline Beds at primary teaching hospital & $447.6(269.7)$ \\
\hline Residency class size & $8.5(3.0)$ \\
\hline
\end{tabular}

* Educational debt categories were based on the ABFM questionnaire's categories.

** Loan repayment program participation was self-reported by respondents to the ABFM questionnaire.

*** Geographic Region was defined based on US Census Bureau regional definitions.

Table 2: Percentage of Graduating Family Medicine Residents Intending to Provide Obstetric Deliveries and Prenatal Care by Resident and Residency Characteristics, 2014-2016 ( N=9,541)

\begin{tabular}{|c|c|c|c|c|c|c|}
\hline \multirow[t]{2}{*}{ Characteristics } & \multicolumn{3}{|c|}{ Obstetric Deliveries } & \multicolumn{3}{|c|}{ Prenatal Care } \\
\hline & Yes & No & & Yes & No & \\
\hline \multicolumn{7}{|l|}{ Individual Characteristics } \\
\hline \multicolumn{7}{|l|}{ Gender } \\
\hline Male & $819(37.9 \%)$ & $\begin{array}{l}3,465 \\
(47.0 \%)\end{array}$ & \multirow{2}{*}{$P<0.01$} & $\begin{array}{l}1,972 \\
(40.4 \%)\end{array}$ & $\begin{array}{l}2,312 \\
(49.7 \%)\end{array}$ & \multirow{2}{*}{$P<0.01$} \\
\hline Female & $\begin{array}{l}1,345 \\
(62.1 \%)\end{array}$ & $\begin{array}{l}3,912 \\
(53.0 \%)\end{array}$ & & $\begin{array}{l}2,912 \\
(59.6 \%)\end{array}$ & $\begin{array}{l}2,345 \\
(50.3 \%)\end{array}$ & \\
\hline \multicolumn{7}{|l|}{ Medical School Type } \\
\hline Osteopathic & $405(18.7 \%)$ & $\begin{array}{l}1,413 \\
(19.2 \%)\end{array}$ & \multirow{2}{*}{$P<0.01$} & $\begin{array}{l}851 \\
(17.4 \%)\end{array}$ & $\begin{array}{l}967 \\
(20.8 \%)\end{array}$ & \multirow{2}{*}{$P<0.01$} \\
\hline Allopathic & $\begin{array}{l}1,759 \\
(81.3 \%)\end{array}$ & $\begin{array}{l}5,964 \\
(80.8 \%) \\
\end{array}$ & & $\begin{array}{l}4,033 \\
(82.6 \%) \\
\end{array}$ & $\begin{array}{l}3,690 \\
(79.2 \%) \\
\end{array}$ & \\
\hline Age (in years) & $32.7(4.3)$ & $32.9(4.4)$ & $P=0.84$ & $32.9(4.4)$ & $32.8(4.3)$ & $P<0.01$ \\
\hline
\end{tabular}


Table 2, continued

\begin{tabular}{|c|c|c|c|c|c|c|}
\hline \multirow[t]{2}{*}{ Characteristics } & \multicolumn{3}{|c|}{ Obstetric Deliveries } & \multicolumn{3}{|c|}{ Prenatal Care } \\
\hline & Yes & No & & Yes & No & \\
\hline \multicolumn{7}{|l|}{ Educational Debt* } \\
\hline Less than $\$ 25,000$ & $380(17.6 \%)$ & $\begin{array}{l}1,589 \\
(21.5 \%)\end{array}$ & \multirow{4}{*}{$P<0.01$} & $\begin{array}{l}1,018 \\
(20.8 \%)\end{array}$ & $\begin{array}{l}951 \\
(20.4 \%)\end{array}$ & \multirow{4}{*}{$P=0.15$} \\
\hline$\$ 25,000-\$ 149,999$ & $479(22.1 \%)$ & $\begin{array}{l}1,237 \\
(16.8 \%)\end{array}$ & & $\begin{array}{l}942 \\
(19.3 \%)\end{array}$ & $\begin{array}{l}774 \\
(16.6 \%)\end{array}$ & \\
\hline$\$ 150,000-\$ 249,999$ & $698(32.3 \%)$ & $\begin{array}{l}2,229 \\
(30.2 \%)\end{array}$ & & $\begin{array}{l}1,513 \\
(31.0 \%)\end{array}$ & $\begin{array}{l}1,414 \\
(30.4 \%)\end{array}$ & \\
\hline$\$ 250,000$ or greater & $607(28.0 \%)$ & $\begin{array}{l}2,322 \\
(31.5 \%)\end{array}$ & & $\begin{array}{l}1,411 \\
(28.9 \%)\end{array}$ & $\begin{array}{l}1,518 \\
(32.6 \%)\end{array}$ & \\
\hline \multicolumn{7}{|c|}{ Loan Repayment Program Participation** } \\
\hline Yes & $513(23.7 \%)$ & $\begin{array}{l}1,301 \\
(17.6 \%)\end{array}$ & \multirow{2}{*}{$P=0.02$} & $\begin{array}{l}1,013 \\
(20.7 \%)\end{array}$ & $\begin{array}{l}801 \\
(17.2 \%)\end{array}$ & \multirow{2}{*}{$P<0.01$} \\
\hline No & $\begin{array}{l}1,651 \\
(76.3 \%)\end{array}$ & $\begin{array}{l}6,076 \\
(82.4 \%)\end{array}$ & & $\begin{array}{l}3,871 \\
(79.3 \%)\end{array}$ & $\begin{array}{l}3,856 \\
(82.8 \%)\end{array}$ & \\
\hline \multicolumn{7}{|l|}{ Residency Program Characteristics } \\
\hline \multicolumn{7}{|l|}{ Geographic Region $* * *$} \\
\hline Northeast (17.6\%) & $265(12.3 \%)$ & $\begin{array}{l}1,395 \\
(19.1 \%)\end{array}$ & \multirow{4}{*}{$P<0.01$} & $\begin{array}{l}822 \\
(16.9 \%)\end{array}$ & $\begin{array}{l}838 \\
(18.2 \%)\end{array}$ & \multirow{4}{*}{$P<0.01$} \\
\hline South $(32.2 \%)$ & $495(23.0 \%)$ & $\begin{array}{l}2,554 \\
(34.9 \%)\end{array}$ & & $\begin{array}{l}1,259 \\
(26.0 \%)\end{array}$ & $\begin{array}{l}1,790 \\
(38.8 \%)\end{array}$ & \\
\hline Midwest (28.4\%) & $682(31.7 \%)$ & \begin{tabular}{|l}
2,002 \\
$(27.4 \%)$
\end{tabular} & & $\begin{array}{l}1,429 \\
(29.4 \%)\end{array}$ & $\begin{array}{l}1,255 \\
(27.2 \%)\end{array}$ & \\
\hline West $(21.8 \%)$ & $711(33.0 \%)$ & $\begin{array}{l}1,357 \\
(18.6 \%)\end{array}$ & & $\begin{array}{l}1,342 \\
(27.7 \%)\end{array}$ & $\begin{array}{l}726 \\
(15.8 \%)\end{array}$ & \\
\hline \multicolumn{7}{|c|}{ Funding as Teaching Health Center } \\
\hline Yes $(4.0 \%)$ & $140(6.5 \%)$ & $242(3.3 \%)$ & \multirow{2}{*}{$P<0.01$} & $\begin{array}{l}240 \\
(5.0 \%)\end{array}$ & $\begin{array}{l}142 \\
(3.1 \%)\end{array}$ & \multirow{2}{*}{$P<0.01$} \\
\hline No $(96.0 \%)$ & $\begin{array}{l}2,013 \\
(93.5 \%)\end{array}$ & $\begin{array}{l}7,066 \\
(96.7 \%)\end{array}$ & & $\begin{array}{l}4,612 \\
(95.0 \%)\end{array}$ & $\begin{array}{l}4,467 \\
(96.9 \%)\end{array}$ & \\
\hline \multicolumn{7}{|c|}{ Federally Qualified Health Center-based } \\
\hline Yes $(21.2 \%)$ & $511(25.6 \%)$ & $\begin{array}{l}1,355 \\
(19.9 \%)\end{array}$ & \multirow{2}{*}{$P=0.03$} & $\begin{array}{l}1,140 \\
(25.5 \%)\end{array}$ & $\begin{array}{l}726 \\
(16.8 \%)\end{array}$ & \multirow{2}{*}{$P<0.01$} \\
\hline No $(78.8 \%)$ & $\begin{array}{l}1,483 \\
(74.4 \%)\end{array}$ & \begin{tabular}{|l}
5,450 \\
$(80.1 \%)$
\end{tabular} & & $\begin{array}{l}3,335 \\
(74.5 \%)\end{array}$ & $\begin{array}{l}3,598 \\
(83.2 \%)\end{array}$ & \\
\hline \multicolumn{7}{|c|}{ Rural Training Track at Residency } \\
\hline Yes $(8.6 \%)$ & $231(10.7 \%)$ & $584(8.0 \%)$ & \multirow{2}{*}{$P<0.01$} & $\begin{array}{l}456 \\
(9.4 \%)\end{array}$ & $\begin{array}{l}359 \\
(7.8 \%)\end{array}$ & \multirow{2}{*}{$P=0.12$} \\
\hline No $(91.4 \%)$ & \begin{tabular}{|l|}
1,922 \\
$(89.3 \%)$
\end{tabular} & \begin{tabular}{|l|}
6,724 \\
$(92.0 \%)$
\end{tabular} & & $\begin{array}{l}4,396 \\
(90.6 \%)\end{array}$ & $\begin{array}{l}4,250 \\
(92.2 \%)\end{array}$ & \\
\hline
\end{tabular}

(continued on next page)

features that contribute to intended scope of practice. Third, many of the individual variables are self-reported by applicants in the ABFM questionnaire. This may lead to respondent bias and also relies on self-interpretation of the potential answer options to questions. Finally, our study only examines associations with intent to practice maternity care, which may not translate into actual practice of maternity care.

\section{Next Steps}

Future research could seek to understand whether this increased intent to provide maternity care will translate into actual practice or if insurmountable barriers exist between the intent to provide and actual practice of maternity care. These data are currently unavailable since the ABFM only started collecting intended scope of practice from graduating residents in 2014. For example, while we know when comparing our study results to the prior study 
Table 2, continued

\begin{tabular}{|c|c|c|c|c|c|c|}
\hline \multicolumn{7}{|c|}{ Residency Program Characteristics } \\
\hline \multirow[t]{2}{*}{ Characteristics } & \multicolumn{3}{|c|}{ Obstetric Deliveries } & \multicolumn{3}{|c|}{ Prenatal Care } \\
\hline & Yes & No & & Yes & No & \\
\hline \multicolumn{7}{|l|}{$\begin{array}{l}\text { Months of Required Maternity Care } \\
\text { Rotations }\end{array}$} \\
\hline $2(44.8 \%)$ & $517(26.4 \%)$ & $\begin{array}{l}3,411 \\
(50.1 \%)\end{array}$ & \multirow{5}{*}{$P<0.01$} & $\begin{array}{l}1,651 \\
(37.2 \%)\end{array}$ & $\begin{array}{l}2,277 \\
(52.6 \%)\end{array}$ & \multirow{5}{*}{$P<0.01$} \\
\hline $3(28.5 \%)$ & $567(28.9 \%)$ & $\begin{array}{l}1,929 \\
(28.3 \%)\end{array}$ & & $\begin{array}{l}1,342 \\
(30.2 \%)\end{array}$ & \begin{tabular}{|l}
1,154 \\
$(26.7 \%)$
\end{tabular} & \\
\hline $4(19.1 \%)$ & $502(25.6 \%)$ & $\begin{array}{l}1,173 \\
(17.2 \%)\end{array}$ & & $\begin{array}{l}966 \\
(21.7 \%)\end{array}$ & \begin{tabular}{|l}
709 \\
$(16.4 \%)$
\end{tabular} & \\
\hline $5(3.9 \%)$ & $148(7.6 \%)$ & $197(2.9 \%)$ & & $\begin{array}{l}219 \\
(4.9 \%)\end{array}$ & \begin{tabular}{|l}
126 \\
$(2.9 \%)$
\end{tabular} & \\
\hline $6(3.7 \%)$ & $226(11.5 \%)$ & $101(1.5 \%)$ & & $\begin{array}{l}265 \\
(6.0 \%)\end{array}$ & $62(1.4 \%)$ & \\
\hline \multicolumn{7}{|c|}{ Maternity Care Fellowship at Residency } \\
\hline Yes $(10.7 \%)$ & $320(16.0 \%)$ & $626(9.1 \%)$ & \multirow{2}{*}{$P<0.01$} & $\begin{array}{l}542 \\
(12.1 \%)\end{array}$ & \begin{tabular}{|l}
404 \\
$(9.3 \%)$
\end{tabular} & \multirow{2}{*}{$P=0.02$} \\
\hline No $(89.3 \%)$ & $\begin{array}{l}1,677 \\
(84.0 \%)\end{array}$ & $\begin{array}{l}6,230 \\
(90.9 \%)\end{array}$ & & $\begin{array}{l}3,957 \\
(87.9 \%)\end{array}$ & $\begin{array}{l}3,950 \\
(90.7 \%)\end{array}$ & \\
\hline Beds at primary teaching hospital & $414.7(284.1)$ & $457.0(264.7)$ & $P<0.01$ & $\begin{array}{l}439.9 \\
(286.8) \\
\end{array}$ & $\begin{array}{l}455.2 \\
(251.2) \\
\end{array}$ & $P=0.13$ \\
\hline Residency class size & $9.1(3.5)$ & $8.4(2.8)$ & $P=0.02$ & $8.8(3.1)$ & $8.2(2.8)$ & $P<0.01$ \\
\hline
\end{tabular}

* Educational debt categories were based on the ABFM questionnaire's categories.

** Loan repayment program participation was self-reported by respondents to the ABFM questionnaire.

*** Geographic region was defined based on US Census Bureau regional definitions.

Figure 1: Proportion of Graduating Family Medicine Residents Intending to Provide Obstetrical Deliveries, by State, 2014-2016
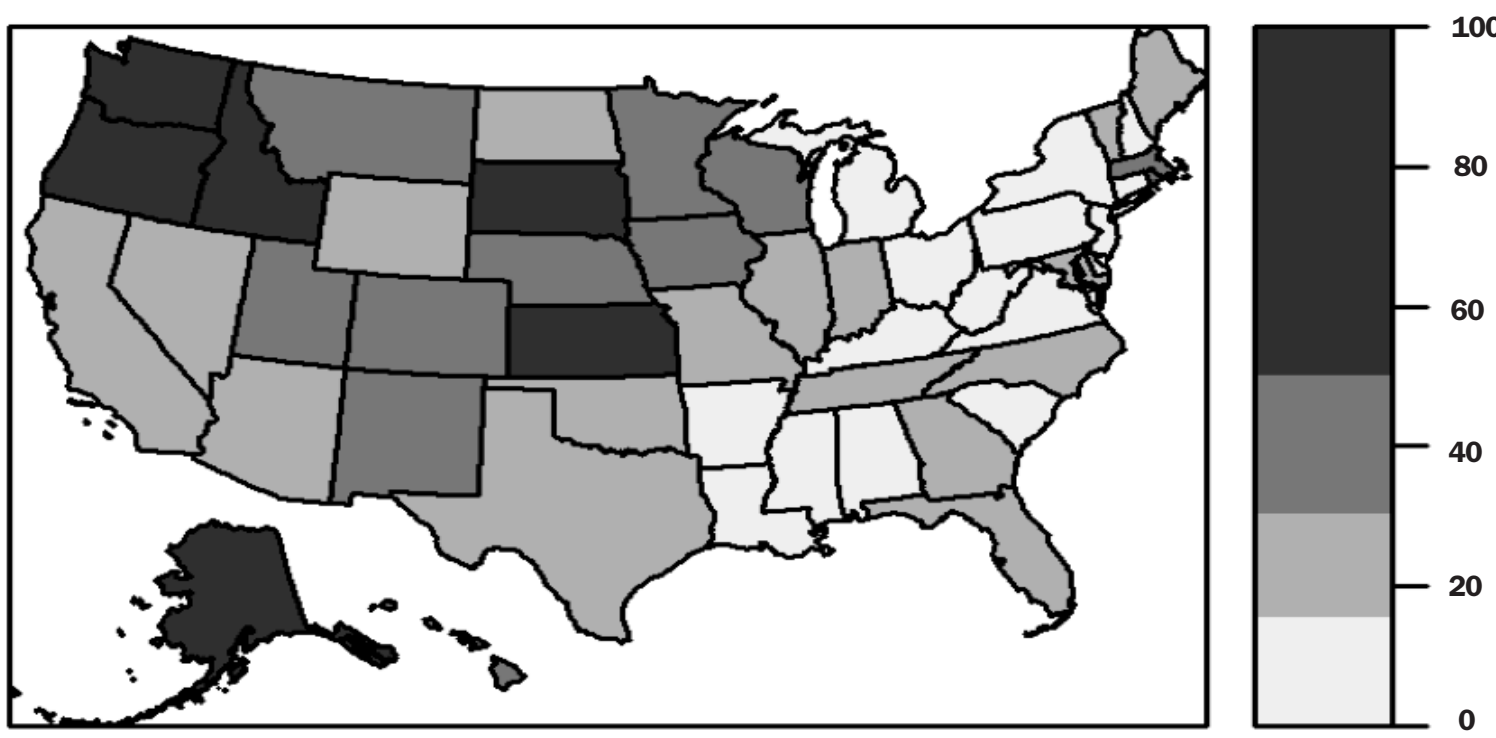

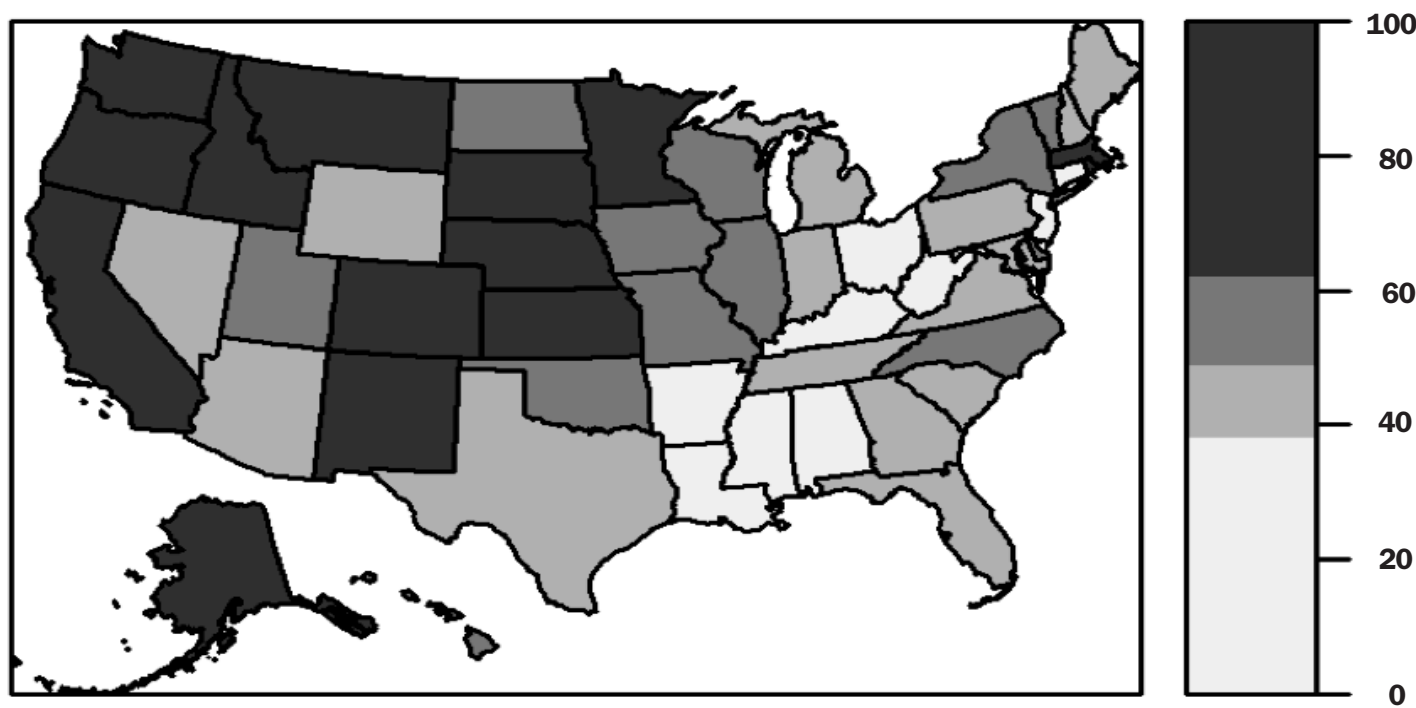

Table 3: Adjusted Significant Associations Between Resident and Residency Characteristics With Resident Intent to Provide Obstetrics or Prenatal Care $(\mathrm{N}=9,541)$

\begin{tabular}{|l|c|c|}
\hline \multicolumn{1}{|c|}{ Individual Characteristics } & $\begin{array}{c}\text { Deliveries Odds } \\
\text { Ratios (95\% Cl) }\end{array}$ & $\begin{array}{c}\text { Prenatal Care Odds } \\
\text { Ratios (95\% Cl) }\end{array}$ \\
\hline Gender (female versus male; ref=male) & & $1.58(1.43,1.73)$ \\
\hline Age (per 1 year age increase) & $--*$ & $1.02(1.01,1.03)$ \\
\hline $\begin{array}{l}\text { Medical school type (allopathic versus osteopathic; } \\
\text { ref=osteopathic) }\end{array}$ & $1.40(1.19,1.64)$ & $1.43(1.26,1.62)$ \\
\hline Loan repayment program participation (ref=no) & $1.22(1.05,1.41)$ & $1.21(1.07,1.37)$ \\
\hline \multicolumn{1}{|c|}{ Residency Program Characteristics } & & \\
\hline Geographic region (ref=West) & & $0.61(0.47,0.80)$ \\
\hline Northeast & $0.55(0.38,0.80)$ & $0.47(0.37,0.60)$ \\
\hline South & $0.58(0.42,0.80)$ & $0.76(0.60,0.96)$ \\
\hline Midwest & $1.06(0.77,1.47)$ & $1.36(1.25,1.48)$ \\
\hline Months of required maternity care rotation (per 1 month increase) & $1.70(1.53,1.90)$ & $--*$ \\
\hline Maternity care fellowship at residency (ref=no) & $1.73(1.19,2.52)$ & $1.51(1.22,1.86)$ \\
\hline Federally Qualified Health Center-based (ref=no) & & $--*$ \\
\hline Teaching Health Center funded (ref=no) & $1.78(1.04,3.04)$ & $-*$ \\
\hline $\begin{array}{l}\text { Number of beds at primary admitting hospital (per } 200 \text { bed } \\
\text { increase) }\end{array}$ & $0.85(0.77,0.93)$ & $-*$ \\
\hline Residency class size (per resident increase) & & $1.04(1.01,1.07)$ \\
\hline
\end{tabular}

CI=confidence interval; ref=reference

* These variables were either not significant in the univariate or multivariate models. 
characterizing family physician provision of maternity care ${ }^{4}$ that intended and actual maternity care practice in the West and Midwest regions is higher, little has been done to elucidate what factors lead to this higher provision in these regions. It is possible that institutional culture, relationships with obstetrician-gynecologists, and legal factors are different in the Midwest and West than in the South and Northeast. To successfully advocate for changes in other regions, understanding these factors will be imperative.

\section{Conclusion}

This study explores, for the first time, the individual and residency program characteristics associated with intent to provide maternity care. Targeted evidence-based policy and programmatic efforts to reauthorize THC funding, support training at FQHCs, increase opportunities for participation in loan repayment programs, increase the number of residencies with maternity care fellowships, and encourage expansion of required maternity care experiences at residencies may help increase resident graduate intent to practice maternity care. If intent to practice maternity care could translate into actual practice, these efforts may increase family medicine scope of practice, increase access to maternity care, decrease costs, and improve health outcomes.
ACKNOWLEDGMENTS: Presented at the North American Primary Care Research Group Annual Meeting on November 14, 2016 in Colorado Springs, CO.

CORRESPONDING AUTHOR: Address correspondence to Dr Tong, Virginia Commonwealth University School of Medicine, 830 E Main Street, Suite 621, PO Box 980101, Richmond, VA, 23298. 804-828-3258. Fax: 804-628-3919. sebastian.tong@vcuhealth.org.

\section{References}

1. Starfield B, Shi L, Macinko J. Contribution of primary care to health systems and health. Milbank Q. 2005;83(3):457-502

2. Grumbach K. To be or not to be comprehensive. Ann Fam Med. 2015;13(3):204-205.

3. Bazemore A, Petterson S, Peterson LE, Phillips RL Jr. More comprehensive care among family physicians is associated with lower costs and fewer hospitalizations. Ann Fam Med. 2015;13(3):206-213

4. Tong ST, Makaroff LA, Xierali IM, Puffer JC, Newton WP, Bazemore AW. Family physicians in the maternity care workforce: factors influencing declining trends. Matern Child Health J. 2013;17(9):1576-1581.

5. Xierali IM, Puffer JC, Tong ST, Bazemore AW, Green LA. The percentage of family physicians attending to women's gender-specific health needs is declining. $J$ Am Board Fam Med. 2012;25(4):406-407.

6. Tong ST, Makaroff LA, Xierali IM, et al. Proportion of family physicians providing maternity care continues to decline. J Am Board Fam Med. 2012;25(3):270-271.

7. Makaroff LA, Xierali IM, Petterson SM, Shipman SA, Puffer JC, Bazemore AW. Factors influencing family physicians' contribution to the child health care workforce. Ann Fam Med. 2014;12(5):427-431.

8. Coutinho AJ, Cochrane A, Stelter K, Phillips RL Jr, Peterson LE. Comparison of intended scope of practice for family medicine residents with reported scope of practice among practicing family physicians. JAMA 2015;314(22):2364-2372.
9. Barreto TW, Eden AR, Petterson S, Bazemore AW, Peterson LE. Intention Versus Reality: Family Medicine Residency Graduates' Intention to Practice Obstetrics. J Am Board Fam Med. 2017;30(4):405-406.

10. Accreditation Council for Graduate Medical Education. ACGME Program Requirements for Graduate Medical Education in Family Medicine. http://www.acgme.org/portals/0/pfassets/programrequirements/120_family_medicine_2017-07-01.pdf. Accessed January 28, 2017.

11. Sutter MB, Prasad R, Roberts MB, Magee SR. Teaching maternity care in family medicine residencies: what factors predict graduate continuation of obstetrics? A 2013 CERA program directors study. Fam Med. 2015;47(6):459-465.

12. American Academy of Family Physicians. Residency Directory. https://nf.aafp.org/Directories/ Residency/Search. Accessed November 6, 2016.

13. Health Resources and Services Administration. Teaching Health Center GME 2014 Grant Awards. http://www.hrsa.gov/about/ news/2014tables/teachinghealthcenters/. Accessed November 6, 2016.

14. The RTT Collaborative. Directory of Rural Programs. https://traindocsrural.org/rural-residency/directory-of-rural-programs/. Accessed November 6, 2016.

15. US Census Bureau. Census Regions and Divisions of the United Stateshttps://www2.census. gov/geo/pdfs/maps-data/maps/reference/us_regdiv.pdf. Accessed November 23, 2017. 